
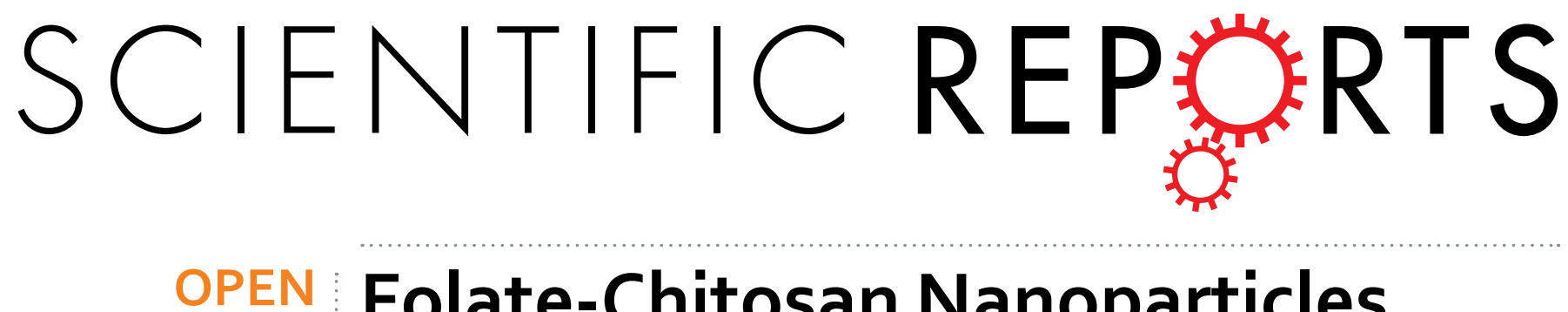

\title{
Folate-Chitosan Nanoparticles Loaded with Ursolic Acid Confer Anti-Breast Cancer Activities
}

Received: 26 March 2015

Accepted: 08 July 2016

Published: 29 July 2016

\section{in vitro and in vivo}

Hua Jin ${ }^{1,2}$, Jiang $\mathrm{Pi}^{1}$, Fen Yang ${ }^{1}$, Jinhuan Jiang ${ }^{1}$, Xiaoping Wang ${ }^{3}$, Haihua Bai ${ }^{4}$, Mingtao Shao ${ }^{3}$, Lei Huang ${ }^{5}$, Haiyan Zhu ${ }^{4}$, Peihui Yang ${ }^{4}$, Lihua $\mathrm{Li}^{4}$, Ting $\mathrm{Li}^{1}$, Jiye Cai ${ }^{1,4}$ \& Zheng W. Chen ${ }^{2}$

Ursolic acid (UA) has proved to have broad-spectrum anti-tumor effects, but its poor water solubility and incompetent targeting property largely limit its clinical application and efficiency. Here, we synthesized a nanoparticle-based drug carrier composed of chitosan, UA and folate (FA-CS-UA-NPs) and demonstrated that FA-CS-UA-NPs could effectively diminish off-target effects and increase local drug concentrations of UA. Using MCF-7 cells as in vitro model for anti-cancer mechanistic studies, we found that FA-CS-UA-NPs could be easily internalized by cancer cells through a folate receptormediated endocytic pathway. FA-CS-UA-NPs entered into lysosome, destructed the permeability of lysosomal membrane, and then got released from lysosomes. Subsequently, FA-CS-UA-NPs localized into mitochondria but not nuclei. The prolonged retention of FA-CS-UA-NPs in mitochondria induced overproduction of ROS and destruction of mitochondrial membrane potential, and resulted in the irreversible apoptosis in cancer cells. In vivo experiments showed that FA-CS-UA-NPs could significantly reduce breast cancer burden in MCF-7 xenograft mouse model. These results suggested that FA-CS-UANPs could further be explored as an anti-cancer drug candidate and that our approach might provide a platform to develop novel anti-cancer drug delivery system.

Cancer remains one of the most devastating diseases threatening public health, causing high mortality worldwide every year. For decades, chemotherapy has served as the preferred treatment. However, conventional chemotherapeutics can't distinguish cancer cells from normal cells, and inevitably damage healthy cells and tissues with evident toxicity. Therefore, it is of central importance to develop efficacious ant-cancer drugs that selectively target cancer cells with low toxicity.

Ursolic acid (UA) is a triterpenoid compound, which exists extensively in food, medicinal herbs, and other plants. Recently, it has been reported that UA can inhibit the growth and development of prostate cancer, liver cancer, and cervical carcinoma ${ }^{1,2}$. Although UA has good anti-cancer activity, clinical application and efficacy of it are still largely limited by its poor water solubility and off-targeting property.

Nanomaterials have recently been emerging as attractive pharmacological vehicles for drug delivery and cancer therapy. The engineered nanomaterials can gain unusual physiochemical characteristics because of their small sizes, surface structure, solubility and shapes. Importantly, nanomaterials can be designed as nanoscale drug carriers to avoid immune clearance by lymphocyte-macrophage system, and therefore allow drugs to efficiently target cancer cells. Over the past decades, inorganic nanoparticles have been explored as drug carriers for new anti-cancer treatments, as nanoparticles can be synthesized to have regular shapes, size, surface chemical and physical properties for better targeting of cancer cells ${ }^{3,4}$. However, inorganic nanoparticles can hardly be degraded in vivo, leading to maintenances in the body for a long time. Moreover, some inorganic nanoparticles have the intrinsic capability to form large aggregates ${ }^{5}$, which result in harmful metabolites and chronic toxicity due to

${ }^{1}$ State Key Laboratory of Quality Research in Chinese Medicines, Macau University of Science and Technology, Macau, 999078, China. ${ }^{2}$ Department of Microbiology and Immunology, University of Illinois, Chicago 60612, USA. ${ }^{3}$ The First Affiliated Hospital of Jinan University, Guangzhou 510632, China. ${ }^{4}$ Department of Chemistry, materials science and engineering, Jinan University, Guangzhou 510632, China. ${ }^{5}$ Treatment and Research Center of Infectious Diseases, the 302 Hospital of PLA, Beijing, 100039, China. Correspondence and requests for materials should be addressed to J.C. (email: tjycai@jnu.edu.cn) or Z.W.C. (email: zchen@uic.edu) 

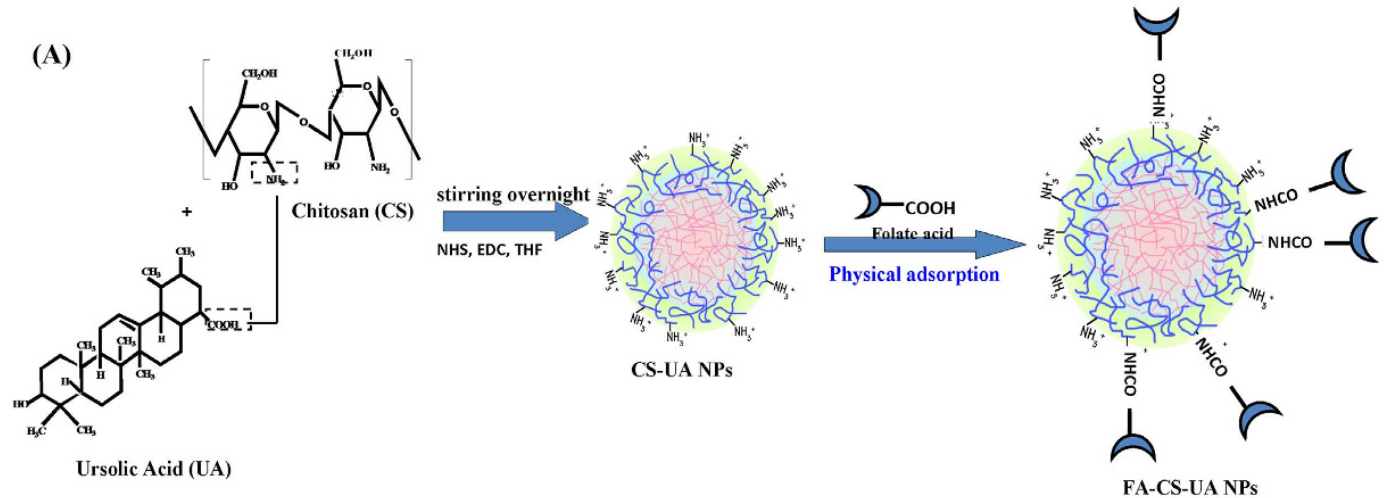

(B)

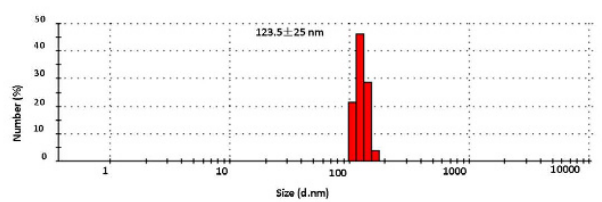

(1).

(D)
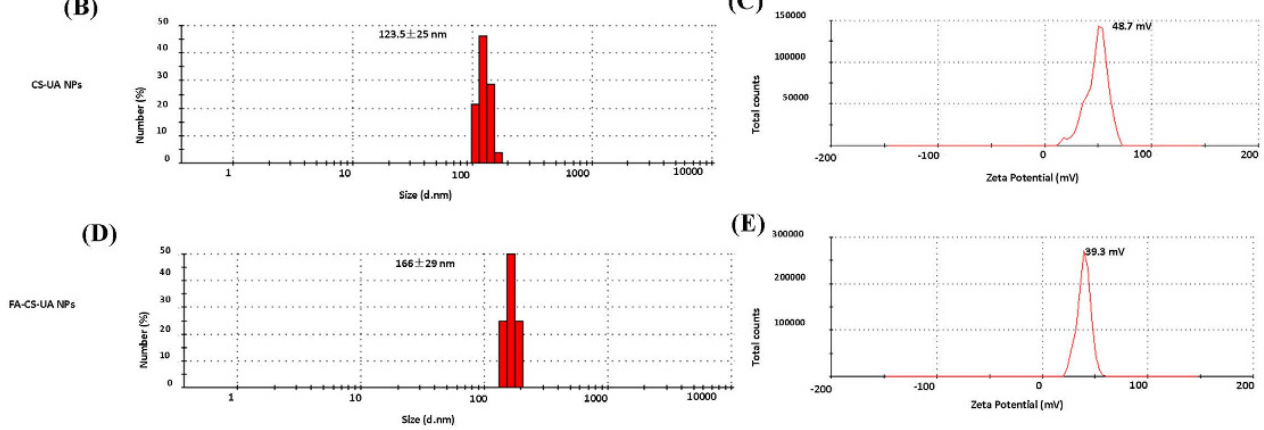

$(\mathbf{E})_{30000}$

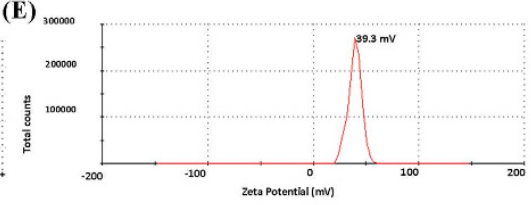

Figure 1. The synthesis and characteristics of folate-coated chitosan nanoparticles loaded UA (FA-CS-UANPs). (A) Illustration of preparation and modification of CS-UA-NPs. (B,D) and (C,E) showed mean sizes and zeta potentials of CS-UA-NPs and FA- CS-UA-NPs, respectively.

redistribution or accumulation in vital organs in the body ${ }^{6}$. Recently, Chan et al. ${ }^{7}$ described the use of DNA to control the biological delivery and elimination of inorganic nanoparticles by organizing them into colloidal superstructures, highlighting new directions in the design of biodegradable and multifunctional nanomedicine.

While nanoparticles have been used to load UA, some methods for synthesis of UA-loaded nanoparticle exhibit low reproducibility ${ }^{8}$ or require tough reaction condition (supercritical anti-solvent process) ${ }^{9}$. Notably, Xiang et al. ${ }^{10}$ encapsulated UA in folate(FA)-targeted liposomes and such formulation largely enhanced the solubility and bioavailability of UA. Sun et al. ${ }^{11}$ developed stably-controlled UA-loaded nanoparticles using amphiphilic mPEG-PCL block copolymers, and demonstrated that such a nano-drug delivery system improved the anti-cancer efficiency of UA leading to more cell apoptosis through stronger inhibition of COX-2 and of caspase-3.

We recently made efforts to design novel biodegradable nanoparticles for loading UA and for improving UA solubility/targeting and anti-cancer efficiency. We employed two innovative approaches in the production of potentially useful UA-loaded nanoparticles. First, we used chitosan (CS) for synthesis of UA-loaded nanoparticles. CS is a linear cationic polysaccharide obtained from natural sources of chitin, such as crabs, krill, and shrimps, which has been regarded as a good candidate for the drug delivery system ${ }^{12,13}$ owing to its excellent biocompatibility, biodegradability and nontoxicity. It actually can protect the drugs from the surrounding medium or low $\mathrm{PH}$ condition under the digestive system through encapsulating active drugs or components in CS polymer micells. Second, we formulated CS, folate(FA), and UA to develop biodegradable nanoparticles (FA-CS-UA-NPs), in which folate molecules were enriched on surface while UAs were encapsulated. Such potentially useful FA-CS-UA-NPs could specifically bind and target folate receptor expressed on cancer cells for delivering UA in the UA-loaded nanoparticles into cells.

We then conducted in vitro and in vivo studies to evaluate novel FA-CS-UA-NPs for the ability to enhance the anti-breast cancer activities and cancer-targeted features or mechanisms. We demonstrated that FA-CS-UA-NPs internalized into cancer cells via folate receptor-mediated pathway and then induced apoptosis in MCF-7 cells through a mitochondria-dependent pathway. Notably, FA-CS-UA-NPs NPs could significantly reduce breast cancer burden in xenograft mouse model. Thus, our approach could provide a platform to design/develop anti-cancer nano-delivery system especially for drugs with poor water solubility.

\section{Results}

Characteristics of FA-CS-UA-NPs. The method for synthesis of FA-CS-UA-NPs was straight forward, and undergoing the mild reaction condition. Figure 1A showed the synthesis process of FA-CS-UA NPs. As shown in Fig. 1B,D, sizes of CS-UA-NPs ranged about $100 \sim 180 \mathrm{~nm}$, with a mean of $122 \mathrm{~nm}$, while the mean size increased to about $160 \mathrm{~nm}$ after folate was conjugated on the surface. Figure S1 (Supporting Information) showed the FA-CS-UA-NPs were in irregular shapes. 

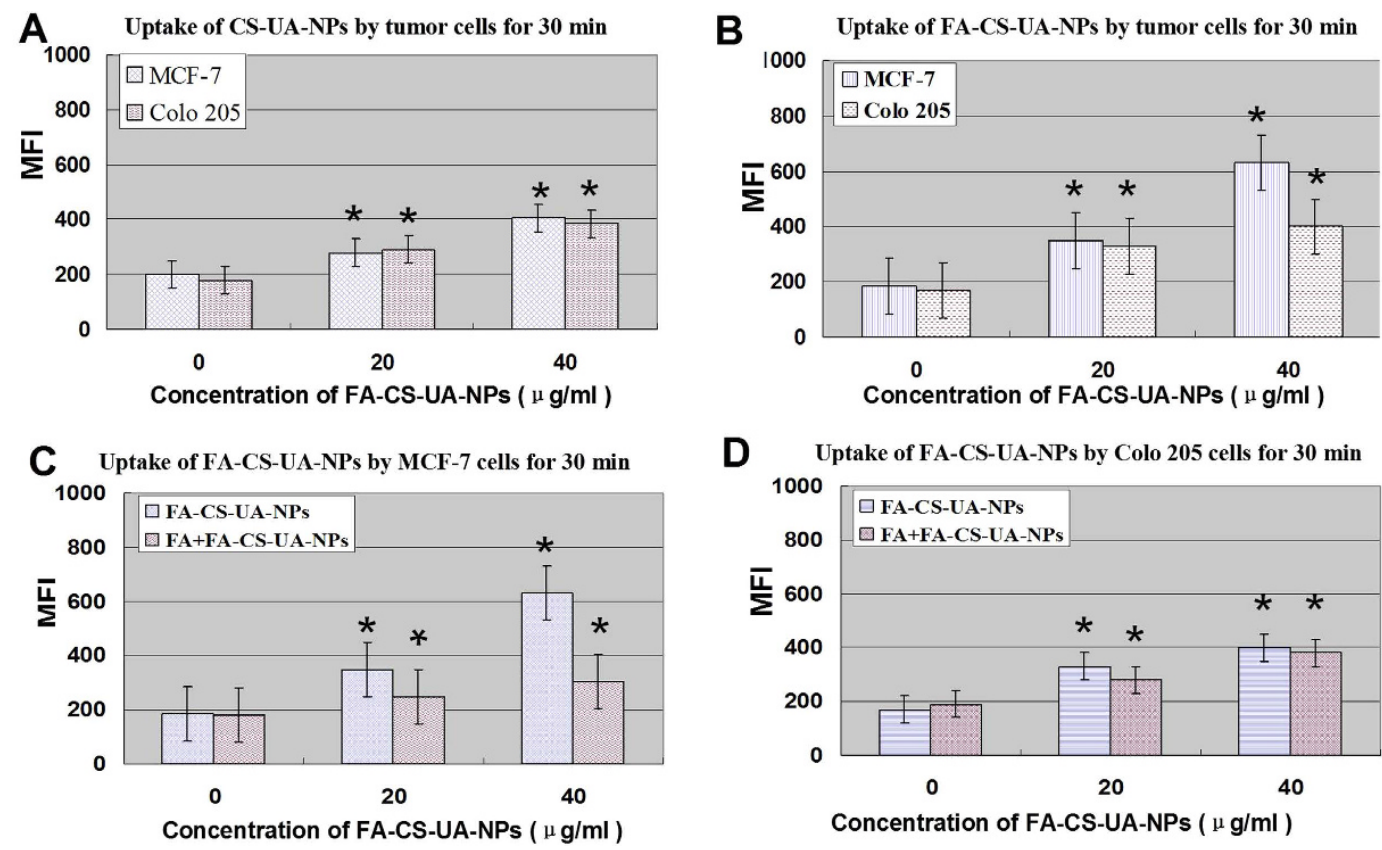

Figure 2. Effects of folate receptors on cellular uptake of FA-CS-UA-NPs. The cellular uptake of CS-UA-NPs (A) and FA-CS-UA-NPs (B-D) by MCF-7 cells and Colo205 cells in the presence and absence $0.1 \mathrm{mM}$ of folate pretreatment for $30 \mathrm{~min}(\mathbf{C}, \mathbf{D})$, respectively. The resulting MFI of RhB loaded in NPs was indicator of the uptake of NPs. ${ }^{*} \mathrm{p}<0.05$ for comparisons between test and control groups in all sub-figures; in Fig. $2 \mathrm{C}, \mathrm{p}<0.05$ is also seen for comparisons between FA + FA-CS-UA-NPs and FA-CS-UA-NPs groups.

Since the electric charges on nanoparticle surface play important roles in physical stability and biocompatibility of nanoparticle-based suspensions, we sought to examine zeta potential of FA-CS-UA-NPs. As shown in Fig. 1C,E, the zeta potential of CS-UA-NPs and FA-CS-UA-NPs was +48.7 and $+39.3 \mathrm{mV}$, respectively. The positive charges of FA-CS-UA-NPs implicated that the amino groups of chitosan were presented on the surface of nanoparticles, and the value of zeta potential $(>25.0 \mathrm{mv})$ implicated that the NPs suspensions were stable and not easy to aggregate.

The HPLC results showed that the drug (UA) loading rate was about $50 \%$.

Cellular uptake of FA-CS-UA-NPs. Many studies ${ }^{14,15}$ have proved that uptake and accumulation of nanomaterials in cells is one of the main factors to generate cytotoxicity. To quantify the uptake level of FA-CS-UA-NPs by cancer cells, fluorescence dye rhodamine-B was encapsulated in the FA-CS-UA-NPs, and the cellular uptake level of FA-CS-UA-NPs was determined by measuring the mean fluorescence intensity (MFI) in cells.

The images obtained from FITC channel by confocal microscopy showed the red fluorescence of the rhodamine B- encapsulated nanoparticles. As shown in Figure S2, increased MFIs in MCF-7 cells were in a dose-dependent manner after 3 hour-treatment with FA- CS-UA-NPs. Most of FA- CS-UA-NPs entered into cells. When MCF-7 cells were incubated with $40 \mu \mathrm{g} / \mathrm{ml}$ FA-CS-UA-NPs for different periods, rhodamine B- encapsulated nanoparticles were still seen in cancer cells 96 hours (Figure S3).

Cellular uptake and endocytic pathways of nanomaterials can critically affect the delivery efficiency and bioavailability of the nano-carrier. There are three main ways of internalizing NPs by cells, i.e. endocytosis, fluid phase endocytosis, and receptor-mediated endocytosis ${ }^{16}$. The HPLC results showed that the concentration of UA in MCF-7 cells after the 24 -hr treatment with the same concentration $(2.0 \mu \mathrm{g} / \mathrm{ml})$ of UA and FA-CS-UA-NPs were 1.1 and $1.4 \mu \mathrm{g} / \mathrm{ml}$, respectively. These results suggested that FA-CS-UA-NPs were much easily internalized by MCF-7 cells.

Given the conjugation of folate on the surface of CS-UA-NPs, we assumed that FA-UA-CS-NPs internalized into cells through folate receptor-mediated endocytosis. To test this hypothesis, MCF-7 cells expressing folate receptors(folate receptor + ) and Colo205 cells expressing no or very low-level folate receptors(folate receptor-) were selected as models, respectively. Fluorescence-based flow cytometry was used to quantitatively measure the uptake level of FA-UA-CS-NPs. To facilitate examination of folate receptor-mediated enodytosis, cells were pretreated with $0.1 \mathrm{mM}$ folate for $1 \mathrm{~h}$ to make sure that the folate receptors on cell membrane was blocked or ligated.

When these two kinds of tumor cells were incubated with non-folate conjugation NPs (CS-UA-NPs) for $30 \mathrm{~min}$ at $37^{\circ} \mathrm{C}$, there were no apparent differences in MFI of CS-UA-NPs between MCF-7 and Colo205 cells (Fig. 2A). In fact, when these two kinds of tumor cells were incubated with folate-conjugated NPs (FA-CS-UA-NPs), there were significant increases in MFI in folate receptor + MCF-7 cells but not folate receptor- Colo205 cells (Fig. 2B), indicating that FA-CS-UA-NPs was more readily uptaken by folate receptor + MCF-7 cancer cells. Moreover, MFI of FA-CS-UA-NPs in control MCF-7 cells not pretreated with folate was remarkably higher than that of MCF-7 cells pretreated with folate (Fig. 2C), suggesting that the cellular uptake of FA-CS-UA-NPs was decreased 
by blocking folate receptors on cell membrane. Consistently, when folate receptor- Colo205 cells were pretreated with free folate molecules and then cultured with FA-CS-UA-NPs, there were no remarkable changes in MFI in colon cells regardless of folate pretreatment (Fig. 2D).

It was likely that when folate receptor $+\mathrm{MCF}-7$ cells were pretreated with free folate molecules, the binding sites of folate receptors on cellular membrane were blocked, leading to significant decreases in the folate receptor-mediated endocytosis of FA-CS-UA-NPs (Fig. 2C). On contrary, folate receptor- Colo205 cells exhibited no significant changes in the cellular uptake of FA-CS-UA-NPs regardless of folate pretreatment, further suggesting that FA-CS-UA-NPs were internalized by cancer cells via a folate receptor-mediated endocytosis pathway.

The quantitative analysis also suggested that FA-UA-CS-NPs were internalized distinctly by two types of cancer cells. Figure S4A also showed that when the concentration of FA-UA-CS-NPs was lower than $40 \mu \mathrm{g} / \mathrm{ml}$, the MFI in two kinds of cells was almost similar. However, when the concentration increased to $80 \mu \mathrm{g} / \mathrm{ml}$, MFI of MCF-7 cell was significantly higher than that of Colo205 cells. Since folate molecules were enriched on the surface, FA-CS-UA-NPs appeared to readily target MCF-7 cancer cells expressing high-level folate receptors. The results supported the point that the cellular uptake was facilitated by a folate receptor-mediated endocytosis pathway.

Figure S4B showed the changes in MFI in cells incubated with the same concentration $(40 \mu \mathrm{g} / \mathrm{ml})$ of FA-UA-CS-NPs at $37^{\circ} \mathrm{C}$ for different time $(0.5,2,3 \mathrm{~h}$, respectively). Increased MFI was seen in cells when the incubation time increases. On the other hand, when the cells were incubated with the same concentration of FA-CS-UA-NPs $(40 \mu \mathrm{g} / \mathrm{ml})$ for the same period $(30 \mathrm{~min})$ in different temperatures, MFI at $37^{\circ} \mathrm{C}$ was significantly higher than that at $4^{\circ} \mathrm{C}$ (Figure S4A,B). These results implied that the cellular uptake of FA-UA-CS-NPs in cancer cells was also in temperature-dependent and time-dependent manner.

Localization of FA-CS-UA-NPs in cells. We then examined the fate of FA-CS-UA-NPs after internalization into cells. To this end, we selected MCF-7 cells as a model to determine the localization of FA-CS-UA-NPs in cells. As shown in Fig. 3A, the red fluorescence represented rhodamine B-associated FA-CS-UA-NPs internalized in MCF-7 cells and the blue fluorescence reflected the nuclei specific marker DAPI. The red fluorescence of FA-CS-UA-NPs was increased in a time-dependent manner, and most of FA-CS-UA-NPs entered into the cytoplasm and circumvented around the nuclei, but no overlay of red and blue color was observed. The results suggested nuclei were not the targets of FA-CS-UA-NPs, and that FA-CS-UA-NPs would not cause genotoxicity in cells during the early stage of FA-CS-UA-NPs incubation.

Effects of FA-UA-CS-NPs on lysosomes and lysosomal membrane integrity. It has been reported that the intracellular localization of nanomaterials closely involved lysosomes and mitochondria ${ }^{17}$ after entry into cells. Here, the distribution of FA-UA-CS-NPs in MCF-7 cells was examined using LysoTracker as a marker of lysosome to investigate the relationship between lysosome locations and FA-UA-CS-NPs. Figure 3B1 showed the co-localization of FA-UA-CS-NPs and lysosomes in MCF-7 cells, and the amounts of FA-UA-CS-NPs located in the lysosomes were increased in a time-dependent manner. The cross line analysis of fluorescence image (Fig. 3B2) also showed the red and green fluorescence were almost entirely coincident.

The integrity of lysosome membrane is important indicator to reflect lysosomal physiological functions. It is well accepted that the long-term accumulation and retention of nanoparticles in lysosomes can influence the lysosomal membrane integrity and further impact their functions. Here, the metachromatic fluorescent cationic dye acridine orange ( $\mathrm{AO}$ ) was used as a probe to detect changes in the integrity of lysosomal membrane induced by as-synthesized nanoparticles. AO could emit strong red light when it accumulates in lysosomes at high concentration, and it will emit green light with weak red fluorescence when it's not concentrated in lysosomes. Nanomaterials such as carbon nanotube, cationic nanoparticles, selenium nanoparticles etc. could destroy the lysosomal membrane and destroy the lysosomal membrane permeabilization (LMP) ${ }^{18}$. As shown in Fig. 3B3, there was enrichment of red fluorescence observed in control MCF-7 cells, but after FA-UA-CS-NPs treatment, MFI of red fluorescence was significantly decreased, indicating the release of $\mathrm{AO}$ from ruptured lysosomes. The results demonstrated that FA-CS-UA-NPs could transport preferentially into lysosomes and then escape from lysosomes and entered into the cytoplasm after destructing the integrity of lysosomal membrane.

Effects of FA-CS-UA-NPs on mitochondria. Next interesting questions would be what would happen and whether FA-CS-UA-NPs could attack and enter into the mitochondria. To address these, Mito Tracker (green color) was used as a mitochondrial fluorescence probe to detect the spatial relationship between FA-CS-UA-NPs and mitochondria in MCF-7 cells. There was only a little overlap of red and green color after $10 \mathrm{~min}$ and $30 \mathrm{~min}$ incubation. However, appreciable overlay of red and green color could be observed after incubation for $60 \mathrm{~min}$ (Fig. 3C1), which was also proved by the cross line analysis (Fig. 3C2). These results suggested FA-CS-UA-NPs entered into mitochondria after being released from lysosomes.

Mitochondria, the energy factories of cells, generate most of ATP that used as a source of chemical energy for cell metabolism. Mitochondria are involved in vital processes of cells, such as molecular signal transduction, cellular differentiation, growth and cell death, etc. As shown in Fig. 3C3, there was a significant decrease in mitochondrial membrane potential $(\Delta \Psi \mathrm{m})$ in MCF-7 cells harboring FA-CS-UA-NPs, implying that FA-CS-UA-NPs could destruct the mitochondrial membrane integrity and induce the collapse of $\triangle \Psi \mathrm{m}$ in MCF-7 cells.

Growth inhibition and apoptosis/necrosis in MCF-7 cells induced by FA-CS-UA-NPs. MTT assay was performed to detect the cell viability of MCF-7 cells after $48 \mathrm{~h}$ treatment with different concentrations of UA and FA-CS-UA-NPs, respectively. As shown in Fig. 4A1, at doses of 40-120 $\mu \mathrm{g} / \mathrm{mL}$, FA-CS-UA-NPs displayed stronger killing effects on MCF-7 cells than UA. For further detection of the death mode of MCF-7, MCF-7 cells were incubated with different concentrations of FA-CS-UA-NPs, and then co-stained by Annexin V-FITC and PI 
(A)
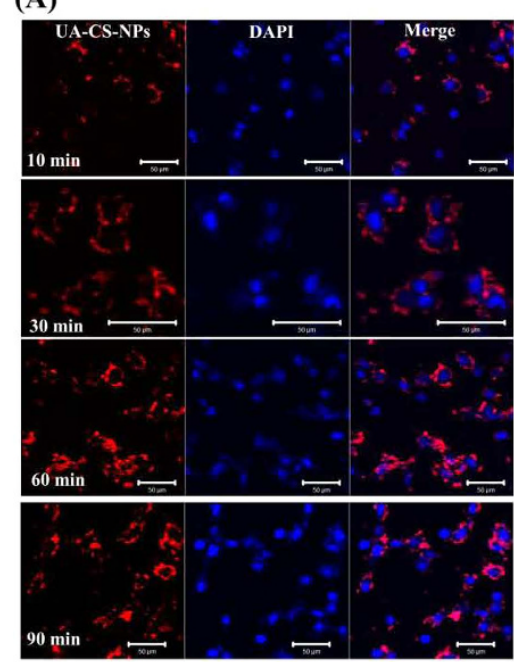

(B1)
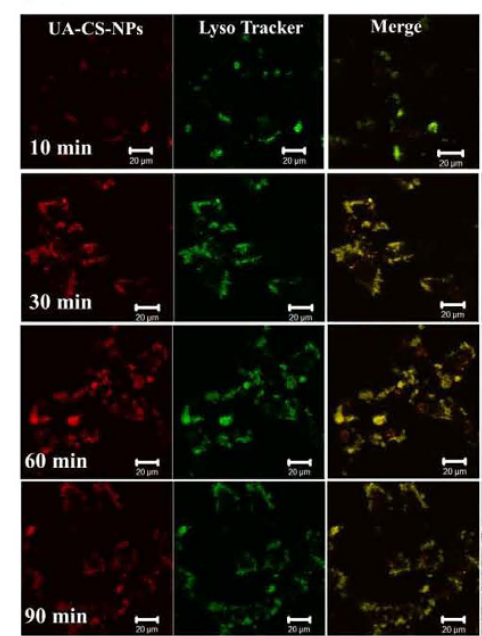

(C1)

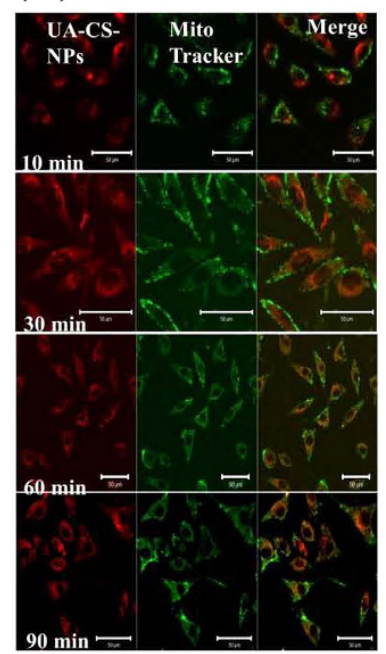

(C2)
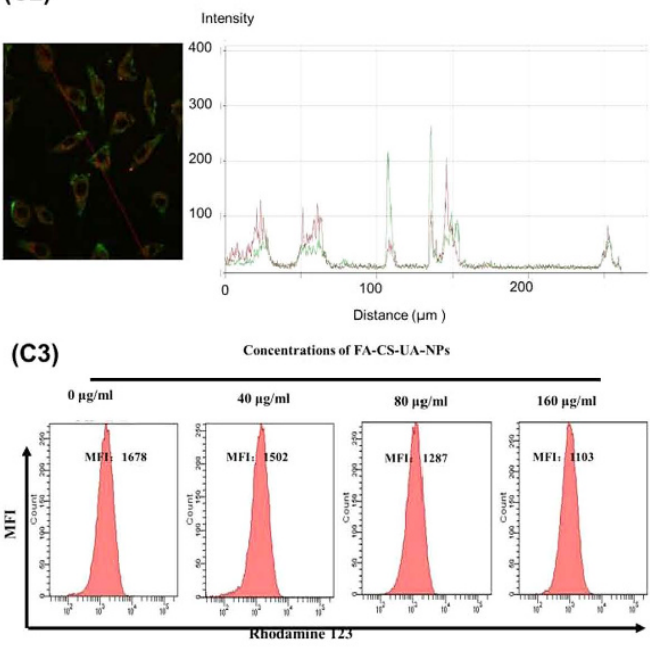

(B2)
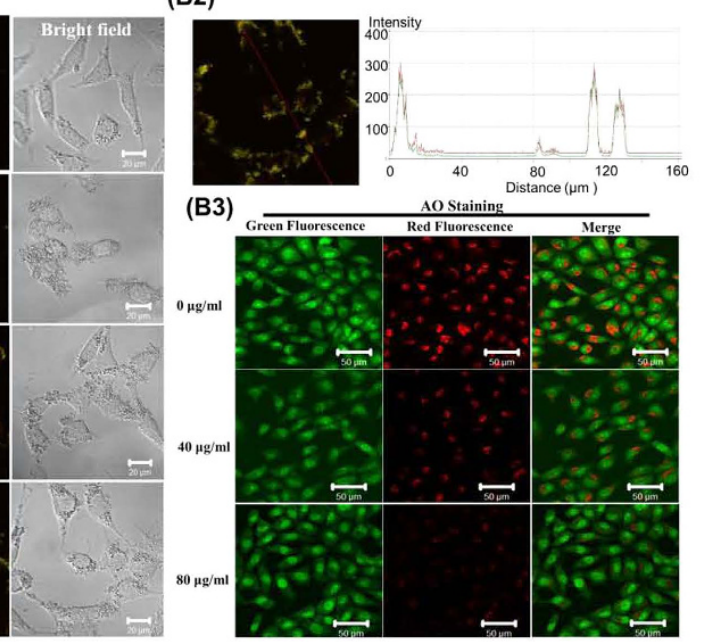

Figure 3. Intracellular trafficking of rhodamine B-loaded FA-CS-UA-NPs after internalized by MCF-7 cells. (A) The FA-CS-UA-NPs (left column), cellular nuclei (middle column), and the merged images demonstrated that nanoparticles (red fluorescence) circumvented around the nuclei (blue fluorescence), but no overlay of the two kinds of fluorescence was observed, suggesting that nuclei were not the cellular targets of FA-CS-UA-NPs. Figure B1-B3 showed the effects of FA-UA-CS-NPs on cellular lysosome: $(B 1, B 2)$ revealed the colocalization of FA-UA-CS-NPs (red) and lysosome (green) in MCF-7 cells after exposure to $20 \mu \mathrm{g} / \mathrm{ml}$ of FA-CS-UA-NPs for different periods of time; (B3) displayed integrity of the lysosomal membrane (AO staining) in MCF-7 cells after cultured with FA-CS-UA-NPs for $6 \mathrm{~h}$, respectively. Scale bar: $20 \mu \mathrm{m}$. Figure C1-C3 showed effects of FA-UA-CS-NPs on mitochondria: (C1) revealed colocalization of FA-UA-CS-NPs (red) and mitochondria (green) in MCF-7 cells after exposure to $40 \mu \mathrm{g} / \mathrm{ml}$ FA- CS-UA- NPs for different periods; (C2) showed the profile indicated the colocalization of FA-UA-CS-NPs and mitochondria; (C3) compared $\Delta \Psi$ in MCF-7 cells after cultured with FA-CS-UA-NPs for $6 \mathrm{~h}$, respectively. Scale bar: $50 \mu \mathrm{m}$.

and measured by flow cytometry. Figure $4 \mathrm{~A} 2$ showed that early apoptosis and necrosis(late apoptosis) appeared to represent the major death mode of MCF-7 cells induced by FA-CS-UA-NPs.

FA-UA-CS-NPs induced ROS overproduction and cell cycle arrest. The intracellular ROS level in MCF-7 cells upon engulfing FA-CS-UA-NPs was investigated by MFI of DCFH. DCFH is formed through intracellular esterase hydrolyzation of a cell absorbed inflorescent probe (DCFH-DA). Figure 4B1 showed that with increasing concentrations of FA-CS-UA-NPs, MFI of DCFH in MCF-7 cells increased significantly, demonstrating the up-regulation of intracellular ROS level upon FA-CS-UA-NPs exposure. Moreover, when we detected the level of ROS generation induced after the different times of FA-CS-UA-NPs treatment, we found that the ROS generation was increased upon FA-CS-UA-NPs exposure from 4 to $12 \mathrm{~h}$, but decreased at the time from 12 to $24 \mathrm{~h}$ of FA-CS-UA-NPs exposure (Figure S5). These results implied that the apoptotic events initiated after FA-CS-UA-NPs treatment for $12 \mathrm{~h}$. To further examine the contribution of ROS generation to FA-CS-UA-NPs-induced apoptosis, MCF-7 cells were co-cultured with FA-CS-UA-NPs and N-acetyl-L-cysteine 


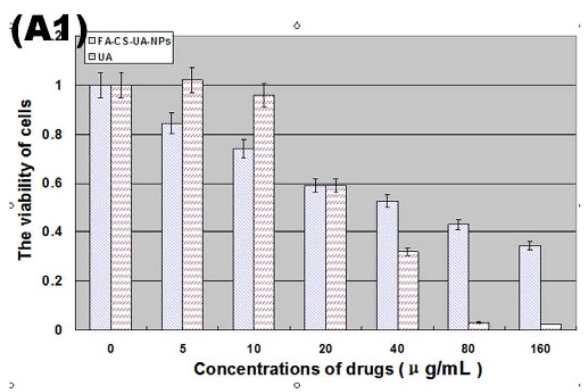

(A2)

Concentrations of UA-CH-NPs
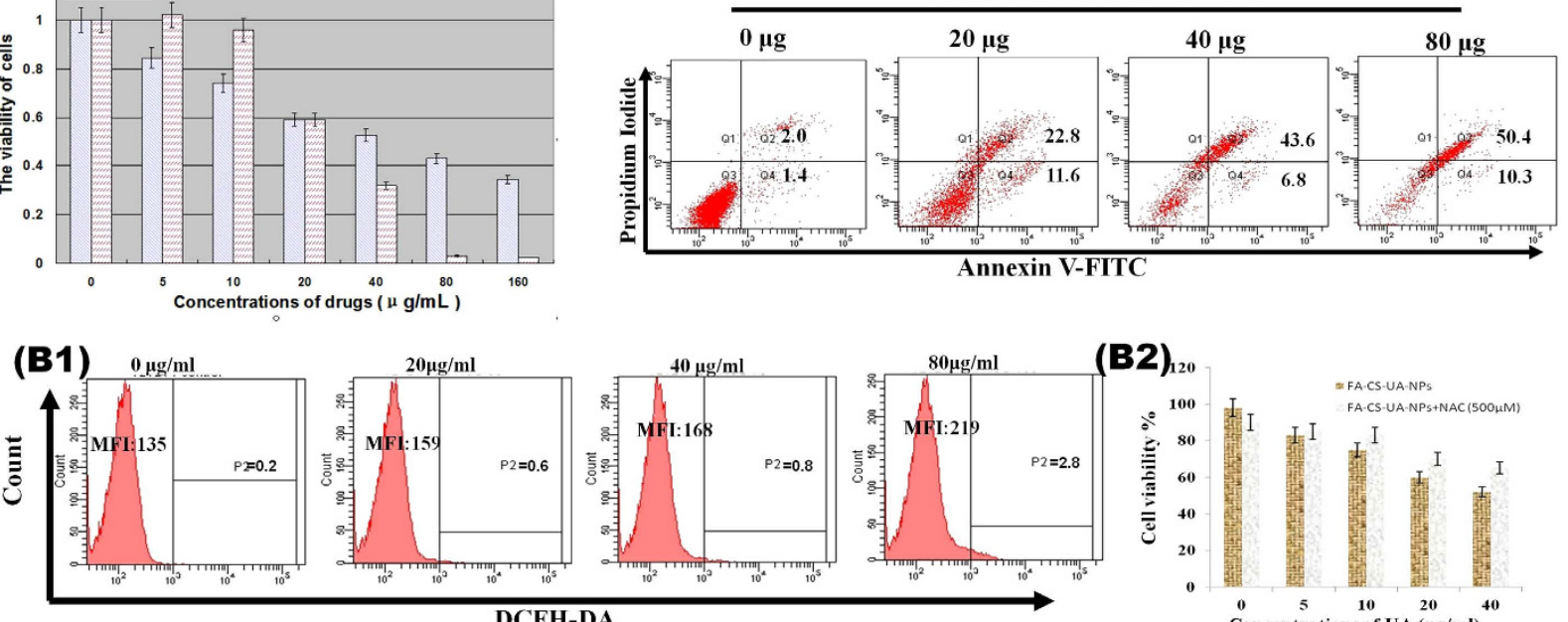

(B2) $)_{120}$
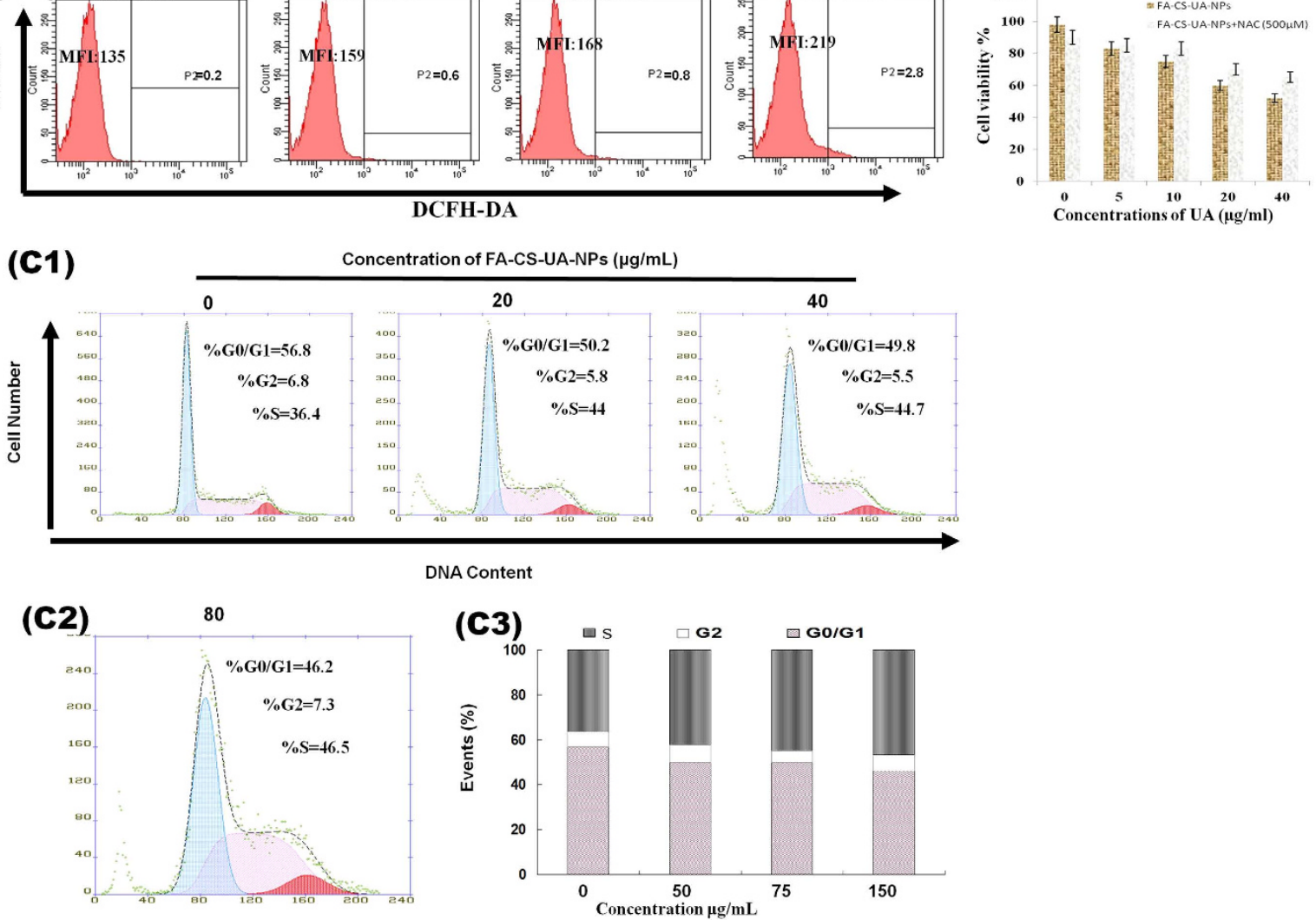

Figure 4. Effects of FA-CS-UA-NPs on cancer cell viability. (A1) Showed comparative viability frequencies of MCF-7 cells treated for $48 \mathrm{~h}$ with different concentrations of UA (hatch bars) and FA-CS-UA-NPs (gray bars).

Note that at doses of $40-120 \mu \mathrm{g} / \mathrm{mL}$, FA-CS-UA-NPs induces significantly greater killing effects on MCF-7 cells than UA ( $\mathrm{P}<0.05$ for comparisons between UA and FA-CS-UA-NPs groups). (A2) Showed representative flow cytometry histograms indicating dose-dependent increases in Annexin V-stained early apoptosis and PI-stained necrosis (late apoptosis) rates of MCF-7 cells induced after 48-hr treatment with different doses of FA-CS-UA-NPs. (B1) Showed that ROS generation in cells was indicated by MFI of DCFH-DA (fluorescent indicator of ROS). (B2) Showed that mean cell viability frequencies of MTT assay evaluating the effects of NAC on cell viability of MCF-7 cells. Cells were pretreated with $500 \mu \mathrm{M}$ NAC for $4 \mathrm{~h}$ and then exposed for $24 \mathrm{~h}$ to FA-CS-UA-NPs. Note that NAC could block ROS production leading to reduction in FA-CS-UA-NPs-mediated killing. (C1-C3) showed the cell cycle alterations in MCF-7 cells treated for $24 \mathrm{~h}$ with different concentrations of FA-CS-UA-NPs. Note a dose-dependent $\mathrm{S}$ phase arrest.

(NAC), the ROS inhibitor, and then assessed for the viability using MTT assay. The results showed that the addition of NAC significantly reduced the ability of FA-CS-UA-NPs to inhibit cellular viability of MCF-7 cells (Fig. 4B2). FA-CS-UA-NPs-induced death in MCF-7 cells appeared to correlate with ROS production (Fig. 4B1,B2).

In parallel, the flow cytometry analysis of PI staining was used to determine the changes in cell cycle distribution induced by FA-CS-UA-NPs. Figure 4C3 showed MCF-7 cells were arrested in S phase, and the cell sub-population in $S$ phase remarkably increased over the increases in the concentrations of FA-CS-UA-NPs (Fig. 4C1-C3). These results suggested that FA-CS-UA-NPs arrested or accumulated MCF-7 cells in DNA synthesis phase, blocked advancing into mitosis phase and ultimately led to the irreversible apoptosis. 


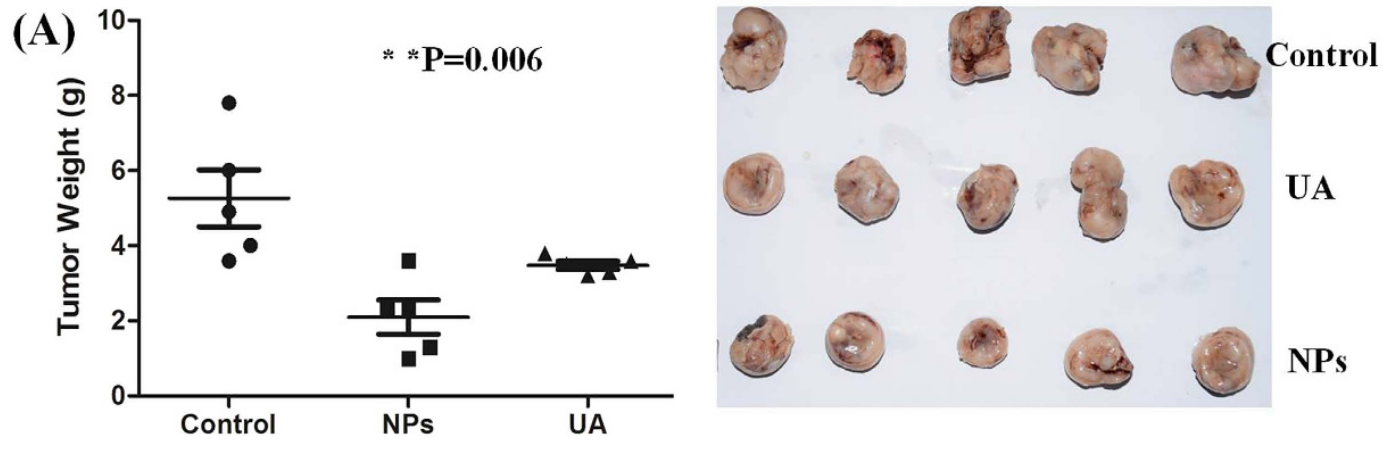

(B)

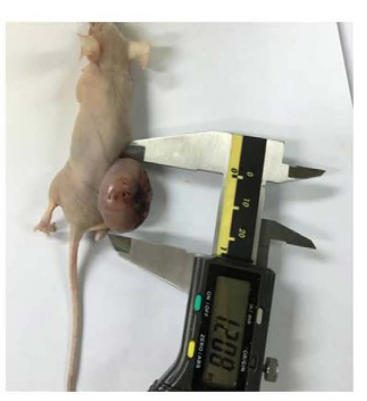

Control

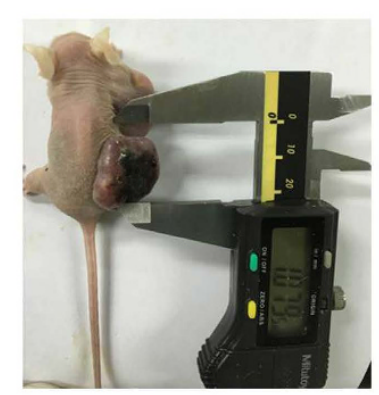

UA

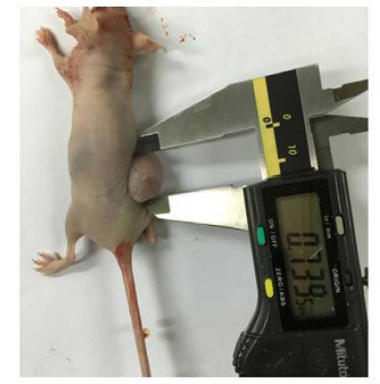

NPs

(C)
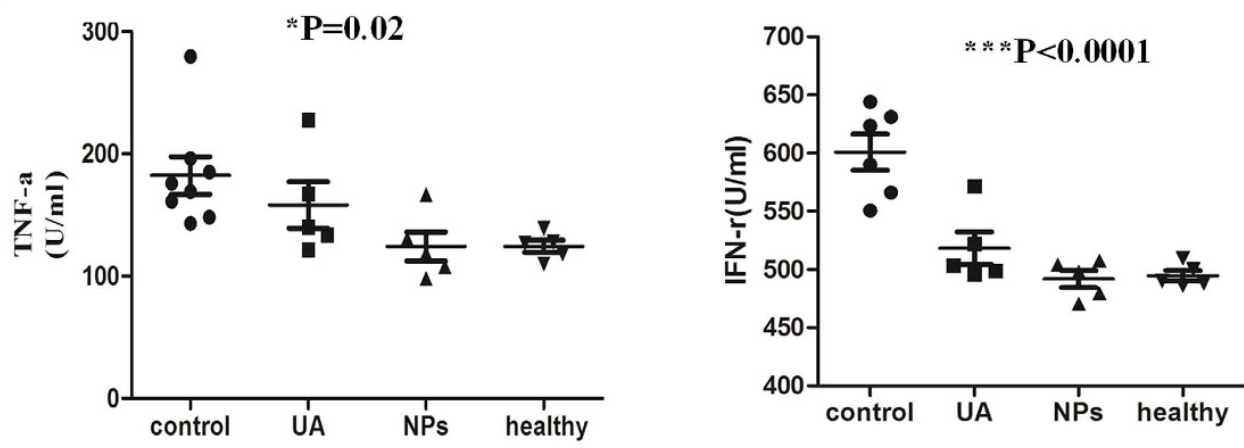

Figure 5. Anti-cancer efficacy of FA-CS-UA-NPs in MCF-7 nude mouse model. (A) Mean weight and images of tumors after i.p. administrated with $0.2 \mathrm{ml}$ of $0.9 \%$ physiological saline (Control group), $12.5 \mathrm{mg} / \mathrm{kg}$ b.w. ursolic acid (UA group) and $12.5 \mathrm{mg} / \mathrm{kg}$ b.w. FA-CS-UA-NPs (NPs group). (B) Representative images of tumor sizes in MCF-7 xenograft mice. (C) ELISA data of serum cytokines (TNF- $\alpha$ and INF-r) in different treated groups. P values indicating statistical significance are derived from ANOVA analysis of control, UA and UA-NPs groups. Nonparametric t test shows similar levels of significance.

FA-CS-UA-NPs-induced anti-cancer activities in vivo. The anti-cancer effects of FA-CS-UA-NPs were determined in MCF-7 cancer xenograft mice. Female Balb/C mice were injected with MCF-7 cells, and at 10 days after injection, the mice were randomly divided into three groups. The control group, positive control group and test group were intraperitoneal injection (i.p.)/day with $0.2 \mathrm{ml}$ of physiological saline, and $12.5 \mathrm{mg} / \mathrm{kg}$ bw of UA and FA-CS-UA-NPs, respectively. Mice were sacrificed and evaluated for changes in cancer burdens after the $8^{\text {th }}$ treatment. As shown in Fig. 5A,B the FA-CS-UA-NPs-treated group exhibited significant decreases in the tumor burden. The tumor weight in FA-CS-UA-NPs-treated group was $2.1 \pm 1.02 \mathrm{~g}$, which was significantly lower than those of UA and saline control groups $(5.26 \pm 1.69 \mathrm{~g}, \mathrm{p}<0.05)$, and UA-treated group $(3.48 \pm 0.24 \mathrm{~g})(\mathrm{P}<0.005)$.

Figure $5 \mathrm{C}$ showed the statistical data of cytokines (IFN- $\gamma$ and TNF- $\alpha$ ) in serum detected by ELISA using ANOVA analysis. After treated with UA or NPs, there was an obvious decrease in expression of IFN- $\gamma$ and TNF- $\alpha$ comparing with saline control group, implying drug-treated group could modify the immune system of tumor-burdened mouse into a good trend.

\section{Discussion}

In the current study, we develop a kind of novel FA-CS-UA-NPs to increase UA solubility, selectively target cancer cells and achieve better therapeutic efficacy against breast cancers in both in vitro and in vivo models. Several studies have assessed high doses of UA for the anti-tumor effects in mouse model. Shanmugam, et al. ${ }^{19}$ reported that 6-week high-dose UA treatment regimen $(200 \mathrm{mg} / \mathrm{kg}$ b.w./every day) inhibited the growth of DU145 cells in 


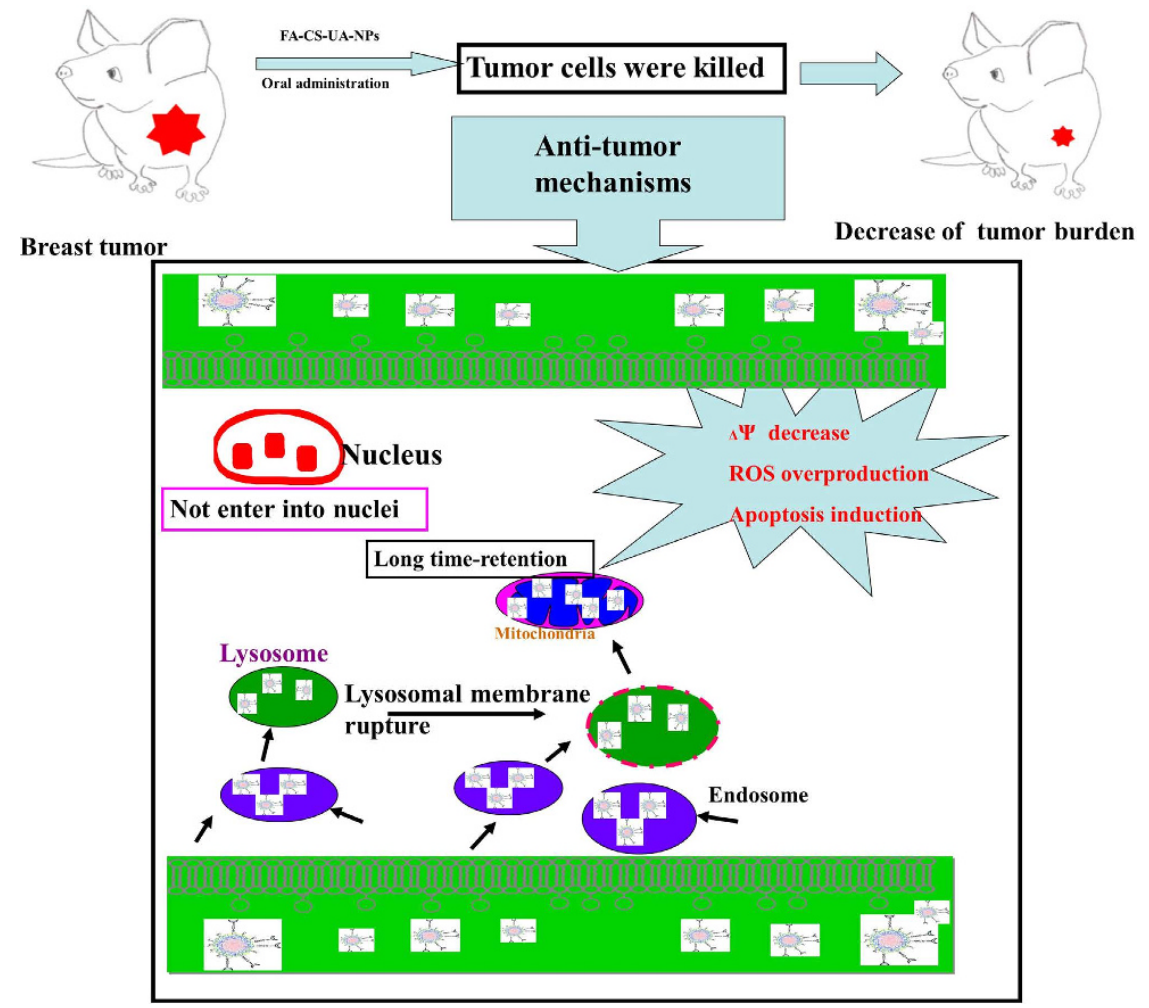

Figure 6. Proposed killing mechanisms of MCF-7 cells induced by FA-CS-UA-NPs.

nude mice. Hursting, et al. ${ }^{20}$ reported UA at $106 \mathrm{mg} / \mathrm{kg}$ b.w./day for 3 weeks was effective against tumor growth in a model of postmenopausal breast cancer. Similarly, Saraswati, et al. ${ }^{21}$ showed that daily treatments with UA at $100 \mathrm{mg} / \mathrm{kg}$ b.w./day dose for 30 days significantly inhibited tumor growth or cell viability in both ascites and solid tumor models. In contrast, our therapeutic UA dose by using the novel FA-CS-UA-NPs is 10 times lower than those reported doses. Such reduced dose and duration for FA-CS-UA-NPs (12.5 mgUA/Kg b.w./day for 10 days) significantly inhibit tumor growth and reduce tumor weights better than UA alone in MCF-7 xenograft nude mouse model. The dramatic improvements for dosing and duration are attributed to our innovative chitosan/ folate nano-carrier system that is able to efficiently carry or deliver UA and selectively target cancer cells, suggesting that FA-CS-UA-NPs delivering UA may significantly reduce or diminish side effects or toxicities potentially induced by high doses of UA or other drugs. Moreover, data of serum cytokines showed that treatments with FA-CS-UA-NPs do not induce apparent pro-inflammatory responses or tissue damages.

The enhanced UA efficacy by using FA-CS-UA-NPs might be explained by two main aspects. First, FA-CS-UA-NPs appear to harness UA pharmacological potency by means of an increased solubility of UA and folate-directed on-target effect. As shown by the MTT results (Fig. 4), FA-CS-UA-NPs more dramatically inhibit MCF-7 cancer cell growth or viability in MCF-7 cancer cells than UA alone. Second, the augmented efficacy of FA-CS-UA-NPs versus UA may also be attributed to the enhanced permeability and retention (EPR) effect, a unique tumors-related phenomenon that can be targeted as a basis for development of macromolecular anti-cancer therapy. Because numerous holes or gaps with 50 500 nanometers exist between endothelial cells in tumor blood vessels and tumor tissues, drug-carriers or macromolecular drugs with sizes of 50 500 nm show selective extravasation and retention and result in prominent anti-tumor effects ${ }^{22}$. Our FA-CS-UA-NPs display sizes about 100-200 nm, and therefore may easily target cancer tissues/cells thanks to the EPR effect in tumor tissues/vessels. FA-CS-UA-NPs with such unique sizes can also enjoy longer half-lives as they are not easily phagocytosed or cleared by macrophages ${ }^{23}$.

FA-CS-UA-NPs appear to have the aggregation-resistant properties in suspension as the high absolute value of zeta potential $(>25 \mathrm{mV})$ has been reported to produce big repulsive electrostatic force between nanoparticles avoiding aggregation. The zeta potential of FA-CS-UA-NPs is $+39.3 \mathrm{mV}$, suggesting that they are stable and could be easily internalized by tumor cells. In addition, due to electrostatic interactions, positively charged FA-CS-UA-NPs can be more easily internalized by cancer cells whose membrane is negatively charged ${ }^{24,25}$.

The current study also provides potential intracellular trafficking mechanisms underlying FA-CS-UA-NPs-mediated killing of cancer cells (Fig. 6). FA-CS-UA-NPs target and enter tumor cells via a folate receptor-mediated endocytosis pathway, which is in energy- and time-dependent fashions. Upon being internalized in lysosomes, FA-CS-UA-NPs appear to destruct permeability and integrity of lysosomal membrane and thereafter get released to the cytoplasm. Then, FA-CS-UA-NPs can enter into mitochondria and induce the overproduction of ROS (Fig. 4B). It has been widely reported that nanomaterials can induce the overproduction of ROS in cancer cells, and such ROS overproduction is responsible for the anti-cancer effect and toxicity of 
nanomaterials. As a natural byproduct of the normal metabolism of oxygen, ROS has important roles in cell signaling and homeostasis ${ }^{26}$. However, dramatic increases of ROS in cells would induce the lipid peroxidation, protein oxidation, mitochondrial damages and other cytotoxic effects, which ultimately led to the collapse of $\Delta \psi$ and even cellular apoptosis. On the other hand, FA-CS-UA-NPs treatments of cancer cells also lead to cell cycle arrest and prevention from entering mitosis phase for cell replication (Fig. 4C). This is consistent with earlier reports suggesting that nano-based materials can cause the DNA damage and chromosomal aberrations and disturbance of cell cycle in the process of cell division ${ }^{27}$.

In summary, we have synthesized a novel nano-carrier using biodegradable chitosan to encapsulate the anticancer drug UA for enhanced water solubility, with folate loaded on surface to improve the UA's targeting and anti-cancer activity. We show that the synthesized nano-drugs, FA-CS-UA-NPs, can stably target cancer cells, induce appreciable anti-cancer activities in breast cancer cells with underlying mechanisms, and confer detectable therapeutic effects against breast cancer in vivo, with no or very low side effects.

\section{Materials}

All chemicals were of analytical grade. UA (98.6\%) and chitosan $(M=1,600)$ were purchased from Shanxi Huike Botanical Development Co., Ltd. (China) and Jinan Haidebei Marine Bioengineering Co. Ltd. (China), respectively. Folate, rhodamine B, Ethyl-(3-3-dimethylam -inopropyl) carbondiimide hydrochloride (EDC), N-hydroxy-succinimide (NHS), 3-(4,5)-dimethylthiahiazo(-z-y1)-3,5-di-phenytetrazoliumromide (MTT), $\mathrm{N}$-acetylcysteine (NAC) were purchased from sigma (USA). Propidium Iodide (PI), Annexin V/PI apoptosis detection kit, $2^{\prime}, 7^{\prime}$-dichlorfluorescein-diacetate(DCFH-DA), 2-(4-Amidinophenyl)-6-indolecarbamidine dihydo-chloride (DAPI), 2-(6-Amino-3-imino-3H-xanthen-9-yl) benzoic acid methyl ester (Rhodamine123), caspase3, caspase 8 and caspase 9 activity assay kit, Mito-Tracker green, Lyso-Tracker red were purchased from Beyotime institute of biotechnology (China). RPMI-1640 medium, DMEM medium, fetal bovine serum (FBS), trypsin and penicillin-streptomycin were purchased from Gibco BRL (USA). Matrigel was obtained from BD biosciences. Milli-Q water was used in all experimental processes. All experimental protocols were approved by IACUC and IBC in accordance with the guidelines of department of Chemistry, the first affiliated hospital of Jinan University, and UI college of Medicine.

Preparation of CS-UA-NPs. $\quad 32 \mathrm{mg}$ of chitosan was dissolved in $5 \mathrm{ml}$ of $1 \%$ (v/v) glacial acetic acid. $10 \mathrm{mg}$ UA, $30 \mathrm{mg}$ EDC and $8 \mathrm{mg}$ NHS were dissolved in $5 \mathrm{ml}$ of THF, after that it was added to the above chitosan solution. Then, the solution was kept on constant magnetic stirring overnight at room temperature. After precipitation with pure water, the solution was centrifuged twice at $11,000 \mathrm{rpm}$ for $20 \mathrm{~min}$ to remove excess amounts of THF and unencapsulated UA. Finally, NPs were lyophilized for 48 hours using lyophilizer for storage in powdered form. To determine the NPs uptake by cells, NPs containing a fluorescent dye (rhodamine B) were prepared using the above procedure, except that $250 \mu \mathrm{g}$ dye was added with the addition of UA to the chitosan solution. The incorporated dye acts as a probe for NPs and offers a sensitive method to determine qualitatively and quantitatively intracellular binding including eventual uptake and retention.

To improve the cell-specific targeting of NPs by cancer cells, the NPs were conjugated with folate to target folate receptor bearing tumor cells. Briefly, the solution of NPs was mixed with folic acid $(0.5 \mathrm{~g} / \mathrm{L})$ for $3 \mathrm{~h}$ at $4{ }^{\circ} \mathrm{C}$, and then centrifuged at $3,000 \mathrm{rpm}$ for $15 \mathrm{~min}$ to remove the unbinded folate.

Characterization of CS-UA-NPs. The size distribution and zeta potential of the nanoparticles was estimated using a Zetasizer Nano ZS (Malvern Instruments, UK). TEM (Philips Co, Holland) and AFM (Veeco, USA) were used to characterize the morphology and dispersed state of the nanoparticles. The micro-graphs were obtained on Hitachi (H-7650) for TEM operated at an accelerating voltage at $80 \mathrm{kV}$. AFM images were achieved by tapping mode in air. A scanning spectro-photometer (Varian, USA) with a 1-cm path length was employed to measure the UV/VIS absorbance of as-prepared products.

To measure the drug (UA) loading rate of FA-CS-UA-NPs, $10 \mathrm{mg}$ lyophilized nanoparticles were dissolved in $1 \mathrm{ml}$ of methanol, and then the amount of UA in the solution was determined by High Pressure Liquid Chromatography (HPLC). HPLC detection was performed using a C18 column $(5 \mu \mathrm{m}, 250 \mathrm{~mm} \times 4.6 \mathrm{~mm})$, whereas the mobile phase, consisting of methanol and $0.1 \%$ acetic acid $(88: 12)(\mathrm{v} / \mathrm{v})$, was maintained at a flow rate of $1.0 \mathrm{~mL} /$ minute. The ultraviolet detector wavelength was $215 \mathrm{~nm}$ and the injection volume was $20 \mu \mathrm{L}$. The drug loading was calculated according to the formula: loading rate $\%=\mathrm{UA} /(\mathrm{CS}+\mathrm{UA}) \times 100 \%$.

To detect the in vitro release rate of Rh B from the NPs, $10 \mathrm{ml}$ of $1 \mathrm{mg} / \mathrm{ml}$ of RhB-loaded NPs were shaken in water bath at $37^{\circ} \mathrm{C}$ at different time points. At each time point, $300 \mu \mathrm{l}$ of samples were taken out and centrifuged. $20 \mu \mathrm{l}$ of supernatant was collected and measured for Rh B concentration in HPLC. HPLC detection was performed using a C18 column $(5 \mu \mathrm{m}, 250 \mathrm{~mm} \times 4.6 \mathrm{~mm})$, whereas the mobile phase, consisting of methanol and $0.1 \%$ acetic acid $(70: 30)(\mathrm{v} / \mathrm{v})$, was maintained at a flow rate of $1.0 \mathrm{~mL} / \mathrm{minute}$. The ultraviolet detector wavelength was $548 \mathrm{~nm}$ of excitation and $578 \mathrm{~nm}$ of emission. Release rate was calculated through dividing supernatant $\mathrm{RhB}$ by total RhB. The release rates of RhB from NPs were $<4 \%$ and $<10 \%$ in 4 and 12 hours, respectively. Figure S8 showed the release of Rhodamine B from the nanoparticles after stirring for different time periods at $37^{\circ} \mathrm{C}$ water bath.

Cell Lines and Cell Culture. MCF-7 breast cancer cells and Colo205 colon adenocarcinoma cells were purchased from American Type Culture Collection (ATCC, USA). All cell lines were grown in DMEM media supplemented with fetal bovine serum (10\%), penicillin ( $100 \mathrm{units} / \mathrm{mL})$, and streptomycin $(100 \mathrm{units} / \mathrm{m} \mathrm{L})$ at $37^{\circ} \mathrm{C}$ in a humidified incubator with $5 \% \mathrm{CO}_{2}$ atmosphere.

Cellular Uptake of FA-CS-UA-NPs. The cellular uptake of FA-CS-UA-NPs was qualitatively detected by fluorescence microscopy. Briefly, treated cells cultured on cover glass in 6-well plates until $70 \%$ confluence were 
incubated with different concentrations of rhodamine B-loaded FA-CS-UA-NPs for various periods of time. The cells were then washed three times by PBS and examined under a fluorescence microscope (Nikon, Japan).

Flow cytometry was used to quantify the cellar uptake of FA-CS-UA-NPs in MCF-7 cells. Briefly, $2 \times 10^{5}$ cells/ well were plated on 6 well plates and incubated $24 \mathrm{~h}$ for attachment. After that, the cells were incubated with designated concentrations of rhodamine B-loaded NPs for different periods. Before analyzed by flow cytometry (BD Inc, USA), the cells were washed triple with PBS and detached by trypsin, washed twice with PBS. The mean fluorescence intensity (MFI) was the indicator of uptake of NPs by cells.

MCF-7 cells $\left(10^{6}\right.$ cells $\left./ \mathrm{ml}\right)$ were cultured with $2 \mu \mathrm{g} / \mathrm{ml}$ (terminal concentration) UA or FA-CS-UA-NPs for $24 \mathrm{~h}$. Then, the cells were washed three times using cold PBS with the supernatants discarded, and then added to the mobile phase, consisting of methanol and $0.1 \%$ acetic acid $(88: 12)(\mathrm{v} / \mathrm{v})$ for HPLC detection. The flow rate was $1.0 \mathrm{~mL} /$ minute. The ultraviolet detector wavelength was $215 \mathrm{~nm}$ and the injection volume was $20 \mu \mathrm{L}$.

MTT assay. Cell viability was determined by measuring the ability of cells to transform MTT to a purple formazan dye. Cells were seeded in 96 -well culture plates at $5 \times 10^{3}$ cells/well for $24 \mathrm{~h}$. The cells were then incubated with FA-CS-UA-NPs at different concentrations for different periods of time. After treatment, $20 \mu \mathrm{L} /$ well of MTT solution $(5 \mathrm{mg} / \mathrm{mL}$ PBS) was added to the well and incubated for another $4 \mathrm{~h}$. For further confirmation the effects of ROS on cell apoptosis, the ROS inhibitor- N-acetyl-L-cysteine (NAC) (2.5 mM), were added into cells $30 \mathrm{~min}$ prior to adding drugs, and then performed MTT assay.

Cell cycle analysis by flow cytometry. MCF-7 cells with or without treatment with FA-CS-UA-NPs for $24 \mathrm{~h}$, were trypsinized, washed twice with PBS, and then, fixed with $70 \%$ chilled ethanol overnight. The fixed cells were washed twice with PBS, treated with RNase A, stained with PI $(50 \mu \mathrm{g} / \mathrm{mL})$, and analyzed by flow cytometer.

Cell apoptosis determination. An Annexin V-FITC/PI apoptosis detection kit was used to detect the apoptotic cells according to the manufacturer's instructions. After treatment with different concentrations of FA-CS-UA-NPs, MCF-7 cells were harvested and washed with cold PBS and suspended in $500 \mu 1$ Annexin V binding buffer. After incubation with $5 \mu \mathrm{l} \mathrm{FITC} \mathrm{labeled-Annexin} \mathrm{V} \mathrm{and} 5 \mu \mathrm{l}$ PI at room temperature in the dark, the samples were immediately analyzed by flow cytometer at $488 \mathrm{~nm}$.

Evaluation of mitochondrial membrane potential $(\Delta \Psi \mathrm{m})$ and intracellular reactive oxygen species (ROS) generation. To detect the changes in $\triangle \Psi \mathrm{m}$ and ROS levels, the MCF-7 cells were treated with different concentrations of FA-CS-UA-NPs and then harvested to incubate with rhodamin 123 and DCFH -DA for $30 \mathrm{~min}$ in dark at room temperature, separately. The cells were collected and washed twice with PBS. The resulting fluorescence was measured by flow cytometer both excited by $488 \mathrm{~nm}$.

In vivo xenograft mouse model. Mice model. MCF-7 xenograft model was established using BALB/c nude mice of $4 \sim 6$ weeks age. The animals were obtained from Beijing HFK Bioscience Co., LTD and quality checks were supervised by Institute of Laboratory of Animal Science (CAM\&PUMC). MCF-7 cells ( $10^{7}$ cells in $50 \mu \mathrm{L}$ serum free medium) with $50 \mathrm{BALB} / \mathrm{c}$ nude mouse of $4 \sim 6$ weeks age matrigel were injected into the back of mouse. The mouse were i.p. administrated with physiological saline, ursolic acid (12.5 mg/kgb.w./day), FA-CS-UA-NPs $(12.5 \mathrm{mg} / \mathrm{kg}$ b.w./day) for nine times. One group of normal healthy mice were used as negative control. There was 5 mice in each group and no mice dead during the whole experimental process.

All experimental protocols were approved by Animal Ethics Committee of Guangdong province, China. And all the experiments were performed in accordance with relevant guidelines and regulations of Animal Ethics Committee of Guangdong province, China.

Measurement of cytokines in blood. The blood of each mouse was collected through the orbital venous, then the serum was collected by centrifugation at $3000 \mathrm{rpm} / \mathrm{min}$ for $10 \mathrm{~min}$. And the serum levels of cytokines (Interferons IFN- $\gamma$, tumor necrosis factor TNF- $\alpha$ ) were determined by ELISA according to the manual instruction of cytokines ELISA kit from R\&D Systems.

Statistical analysis. Statistical analysis was performed using nonparametric t test or ANOVA test, with $* \mathrm{P}<0.05$ regarded as statistically significant.

\section{References}

1. Zhang, Y. X. et al. Ursolic Acid Overcomes Bcl-2-Mediated Resistance to Apoptosis in Prostate Cancer Cells Involving Activation of JNK-Induced Bcl-2 Phosphorylation and Degradation. J Cellular Biochem 109, 764-773 (2010).

2. Yim, E. K. et al. Anti-proliferative and antiviral mechanisms of ursolic acid and dexamethasone in cervical carcinoma cell lines. Int J Gynecol Cancer 16, 2023-2031 (2006).

3. Bisker, G. et al. Controlled release of Rituximab from gold nanoparticles for phototherapy of malignant cells. J Control Release 162, 303-309 (2012)

4. Guo, Z. R. et al. Light-Switchable Single-Walled Carbon Nanotubes Based on Host-Guest Chemistry. Adv Funct Mater 23, 5010-5018 (2013).

5. Albanese, A. \& Chan, W. C. Effect of Gold Nanoparticle Aggregation on Cell Uptake and Toxicity. ACS Nano 5, 5478-5489 (2011).

6. Ye, L. et al. A pilot study in non-human primates shows no adverse response to intravenous injection of quantum dots. Nat Nanotechnol 7, 453-458 (2012).

7. Chou, L. Y., Zagorovsky, K. \& Chan, W. C. DNA assembly of nanoparticle superstructures for controlled biological delivery and elimination. Nat Nanotechnol 9, 148-155 (2014).

8. Gao, D., Tang, S. \& Tong, Q. Oleanolic acid liposomes with polyethylene glycol modification: promising antitumor drug delivery. Int of Nanomedicine 7, 3517-3526 (2012).

9. Yang, L. et al. Physicochemical properties and oral bioavailability of ursolic acid nanoparticles using supercritical anti-solvent (SAS) process. Food Chemistry 132, 319-325 (2012). 
10. Yang G. et al. In vitro and in vivo antitumor effects of folate-targeted ursolic acid stealth liposome. J Agric Food Chem. 62, 2207-2215 (2014).

11. Zhang H. et al. Delivery of ursolic acid (UA) in polymeric nanoparticles effectively promotes the apoptosis of gastric cancer cells through enhanced inhibition of cyclooxygenase 2 (COX-2). Int J Pharm. 441, 261-268 (2013).

12. Nam, H. Y. et al. Cellular uptake mechanism and intracellular fate of hydrophobically modified glycol chitosan nanoparticles. J Control Release 135, 259-267 (2009).

13. Koo, H. et al. Enhanced drug-loading and therapeutic efficacy of hydrotropic oligomer-conjugated glycol chitosan nanoparticles for tumor-targeted paclitaxel delivery. J Control Release 172, 823-831 (2013).

14. Wang, L. et al. Selective targeting of gold nanorods at the mitochondria of cancer cells: implications for cancer therapy. Nano Lett 9 , 772-780 (2011).

15. Liu, W. et al. Selenium Nanoparticles as a Carrier of 5-Fluorouracil to Achieve Anticancer Synergism. ACS Nano 6, 6578-6591 (2012).

16. Yang, P. H. et al. Transferrin-mediated gold nanoparticle cellular uptake. Bioconjug Chem 16, 494-496 (2005).

17. Zhao, F. et al. Cellular uptake, intracellular trafficking, and cytotoxicity of nanomaterials. Small 7, 1322-1337 (2011).

18. Xia, T. et al. Cationic polystyrene nanosphere toxicity depends on cell-specific endocytic and mitochondrial injury pathways. ACS Nano 2, 85-96 (2008).

19. Shanmugam, M. K. et al. Ursolic acid inhibits multiple cell survival pathways leading to suppression of growth of prostate cancer xenograft in nude mice. J Mol Med 89, 713-727 (2011).

20. De Angel, R. E. et al. Antitumor Effects of Ursolic Acid in a Mouse Model of Postmenopausal Breast Cancer. Nutr Cancer 62, 1074-1086 (2010).

21. Saraswati, S., Agrawal, S. S. \& Alhaider, A. A. Ursolic acid inhibits tumor angiogenesis and induces apoptosis through mitochondrialdependent pathway in Ehrlich ascites carcinoma tumor. Chem Biol Interact 206, 153-165 (2013).

22. Choi, H. S. et al. Renal clearance of quantum dots. Nat biotechnol 25, 1165-1170 (2007).

23. Makino, K. et al. Phagocytic uptake of polystyrene microspheres by alveolar macrophages: effects of the size and surface properties of the microspheres. Colloids Surf. B Biointerfaces 27, 33-39 (2003).

24. Asati, A. et al. Surface-charge-dependent cell localization and cytotoxicity of cerium oxide nanoparticles. ACS Nano 4, 5321-5331 (2010).

25. Slowing, L., Trewyn, B. G. \& Lin, V. S. Effect of Surface Functionalization of MCM-41-Type Mesoporous Silica Nanoparticles on the Endocytosis by Human Cancer Cells. J Am Chem Soc 128, 14792-14793 (2006).

26. Koppolu, B. \& Zaharoff, D. A. The effect of antigen encapsulation in chitosan particles on uptake, activation and presentation by antigen presenting cells. Biomaterials 34, 2359-2369 (2013).

27. Wilhelm, C. et al. Interaction of anionic super paramagnetic nanoparticles with cells: Kinetic analyses of membrane adsorption and subsequent internalization. Langmuir 18, 8148-81559 (2002).

\section{Acknowledgements}

This work was financially supported by Macao Science and Technology Development Fund (No. 028/2014/A1), the Overseas, Hong Kong \& Macao Cooperative Research Funds of China (No. 31129002) and Jinan University's Scientific Research Cultivation and Innovation Fund (No. 21612601). H.J., J.C. L.S. and Z.W.C are supported in part by the NIH RO1 grants (HL64560/ OD015092/AI106590/HL129887).

\section{Author Contributions}

H.J. designed and performed the experiments, interpreted the data and drafted the manuscript, F.Y. and J.P. conceived of the study, participated in its design and helped to draft the manuscript, J.J., X.W., M.S., P.Y., H.Z., T.L. and L.L., conceived of the study, participated in its design and coordination, J.C. supervised all experimental procedures and helped to draft the manuscript, Z.W.C. evaluated and discussed experiments and data, and helped to write and revised manuscript. All authors reviewed the manuscript.

\section{Additional Information}

Supplementary information accompanies this paper at http://www.nature.com/srep

Competing financial interests: The authors declare no competing financial interests.

How to cite this article: Jin, H. et al. Folate-Chitosan Nanoparticles Loaded with Ursolic Acid Confer AntiBreast Cancer Activities in vitro and in vivo. Sci. Rep. 6, 30782; doi: 10.1038/srep30782 (2016).

(c) (i) This work is licensed under a Creative Commons Attribution 4.0 International License. The images or other third party material in this article are included in the article's Creative Commons license, unless indicated otherwise in the credit line; if the material is not included under the Creative Commons license, users will need to obtain permission from the license holder to reproduce the material. To view a copy of this license, visit http://creativecommons.org/licenses/by/4.0/

(c) The Author(s) 2016 